\title{
Human menstrual blood-derived mesenchymal stem cells regulation of the EGF/Ras p21 pathway as a potential therapeutic target for thin endometrium
}

\author{
Mei Zhao", Fengli Chi", Tingyu Zhang, Xiaoming Teng, Kunming Li \\ Department of Assisted Reproductive Medicine, Shanghai First Maternity and Infant Hospital, School of Medicine, Tongji University, Shanghai, \\ China \\ Contributions: (I) Conception and design: X Teng, M Zhao, F Chi; (II) Administrative support: K Li; (III) Provision of study materials or patients: All \\ authors; (IV) Collection and assembly of data: All authors; (V) Data analysis and interpretation: All authors; (VI) Manuscript writing: All authors; (VII) \\ Final approval of manuscript: All authors. \\ \#These authors contributed equally to this work. \\ Correspondence to: Kunming Li; Xiaoming Teng. Department of Assisted Reproductive Medicine, Shanghai First Maternity and Infant Hospital, School \\ of Medicine, Tongji University, 2699 West Gaoke Road, Shanghai 200040, China. Email: likunming@hotmail.com; tengxiaoming@hotmail.com.
}

Background: Human infertility is caused by many factors, among which thin endometrium is the main
reason for poor embryo implantation. Currently, stem cell therapy could be a potential approach in treating
human endometrial disorder like thin endometrium. In this study, we aimed to explore the influence of
menstrual stem cells from non-thin endometrium (NTE-MenSCs) and thin endometrium (TE-MenSCs) on
the phenotype of endometrial epithelial cells (EECs).

Methods: The MenSCs were isolated from women with and without thin endometria, characterized and co-cultured with the EECs. The expression of cytokeratin 7 (CK7) was verified by immunofluorescence while the detection stem cell markers was determined flow cytometry. Osteogenic and adipogenic differentiation were induced in appropriate media. The quantitative real-time PCR and western blotting were respectively used for detecting the mRNA and protein expression levels, respectively. The CCK- 8 assay was used for cell viability analysis whereas ELISA was used for the detection of cytokine levels.

Results: The results showed that the co-culture of NTE-MenSCs or TE-MenSCs and EECs promoted the proliferation, migration, and angiogenesis of endothelial progenitor cells differently. Furthermore, the TE-MenSCs promoted the expression of inflammation, vascularized adipose, and extracellular matrix related proteins. The epidermal growth factor (EGF)/Ras p21 pathway was found to mediate the influence of MenSCs on EECs.

Conclusions: These findings are vital in that they may promote stem cell therapy of thin endometrium and enable embryo implantation in humans with thin endometrium.

Keywords: Thin endometrium; menstrual stem cells; endometrial epithelial cells (EECs); embryo implantation

Submitted Aug 10, 2021. Accepted for publication Sep 18, 2021.

doi: $10.21037 / \mathrm{atm}-21-4652$

View this article at: https://dx.doi.org/10.21037/atm-21-4652

\section{Introduction}

Female infertility is a major public health problem of multiple etiologies and its management constitutes an economic, psychological, and social burden for infertile patients; the prevalence of female infertility is increasing and, until today, no effective treatment for infertility has been developed (1-3). Hormonal treatments, in vitro fertilization and embryo transfer remain the only options for infertile patients who wish to have children. Embryo transfer technology, despite being more effective than other 
therapeutic options, is challenged by the low implantation rate (4-7), the success of which depends on the quality of the embryo and the endometrial receptivity (8).

The thickness of the endometrium is an important factor affecting the receptivity of the endometrium $(9,10)$. A thin endometrium is characterized as an endometrium with thickness lower than $7 \mathrm{~mm}$ on ultrasound (11). The causes of thin endometrium are multifactorial with the most incriminated factors being inflammation, drugs, poor vascularization and low estradiol levels (11). The thin endometrium is a critical factor that limits embryo implantation and the success in vitro fertilization-embryo transfer $(8,12,13)$. The therapeutic options for thin endometrium mainly include drug treatments and stem cell therapy, among others, but these options currently remain ineffective (9,14-17). Recent studies have shown that stem cells can migrate to the endometrium, increase endometrial thickness, and improve endometrial receptivity, which provides new avenues for developing treatments for thin endometrium (18-20).

Mesenchymal stem cells are a type of pluripotent stem cell belonging to the mesoderm and mainly exist in the interstitium and connective tissues of organs $(21,22)$. In recent years, a new type of mesenchymal stem cell has been discovered, isolated, and extracted, known as menstrual blood-derived mesenchymal stem cells (MenSCs) (23). The MenSCs are adult stem cells, which are capable of long-term self-replication, enabling them to play a role in regenerative medicine, especially as a tool for stem cell therapy (23). They can also express some of the surface markers of embryonic stem cells, colonize the injury site through homing, express and secrete various cytokines, and participate in tissue repair and regeneration (24-26). MenSCs are positive for markers such as HLAABC, OCT-4, CD73, CD90, SSEA-4, CD49a and CD105 but negative for HLA-DR and hematopoietic lineage markers STRO-1 $(23,25,26)$. However, inconsistencies in MenSCs phenotype have been reported for markers c-KIT and SSEA-4 $(23,25,26)$. The MenSCs are considered an ideal seed cell for the treatment of thin endometrium, which inhibits embryo implantation (24-26). A previous study indicated that MenSCs not only contribute to the repair of endometrium via upregulating VEGF, vimentin and keratin, but also increase the fertility and embryo implantation rate of injured endometrium (27). Another study indicated that tail vein injection of MenSCs promotes endometrial regeneration via regulating the FGF2 protein (28). However, whether the interactions of MenSCs with endometrial cells, especially endometrial epithelial cells (EECs) play a potential role in the the treatment of thin endometrium and the underlying mechanism are not clear.

Therefore, we aimed to study the effects of MenSCs on the phenotypes of endometrial epithelial cells (EECs) and the corresponding mechanism. These results can provide a theoretical basis for the future clinical application of MenSCs, thereby opening up new ideas for improving the clinical outcome of human assisted reproductive technology. We present the following article in accordance with the MDAR reporting checklist (available at https://dx.doi. org/10.21037/atm-21-4652).

\section{Methods}

\section{Reagents}

The antibodies used for flow cytometry included: monoclonal antibodies conjugated to FITC-A against CD9 (Cat \#555371), CD105 (Cat \#561443), as well as monoclonal antibodies conjugated to APC for CD44 (Cat \#560532); monoclonal antibodies conjugated to PECy7-A for CD34 (Cat \#550761) and CD38 (Cat \#560677), monoclonal antibodies conjugated to PE-A for CD72 (Cat \#316207) and SSEA4 (Cat \#330405). The antibodies CD9, CD105, CD44, CD34, CD38, and the corresponding control antibodies (isotypes) were purchased from BD Biosciences (Becton, Dickinson, and Co. Biosciences, San Jose, CA, USA), CD72 and SSEA4 were purchased from Biolegend (Biolegend, San Diego, CA, USA), All of the antibodies used in flow cytometry experiments were based on the concentrations recommended by the manufacturer's manual.

The antibodies used for the western blot experiments were the primary antibodies against FGF (Cat \#AF-233SP), MMP3 (Cat \#MAB513-SP), platelet-derived growth factor (PDGF; Cat \#AF385-SP), EGF (Cat \#AF236), tumor necrosis factor- $\alpha$ (TNF- $\alpha$; Cat \#MAB610-SP) and $\beta$-Actin (Cat \#MAB8969); these antibodies were acquired from R\&D Biosystems (Minneapolis, MN, USA) while the antibody conjugated to peroxidase was used as a secondary antibody.

\section{Patient selection}

This study was approved by the Institutional Review Board and the Ethics Committee of Tongji University (2019tjdx352). All procedures performed in this study 
involving human participants were in accordance with the Declaration of Helsinki (as revised in 2013). The membership census took place between 2017 and 2019 at Shanghai First Maternity and Infant Hospital, and all participants had signed an informed consent form before the start of the study. Women with a "thin" endometrium ( $\mathrm{n}=10$, mean age $28 \pm 2$, endometrial thickness $<7 \mathrm{~mm}$ ) hospitalized for laparoscopy for infertility were included in this study. The control group included women with a normal (nonthin) endometrium $(n=10$, mean age $29 \pm 6$, endometrial thickness $\geq 7 \mathrm{~mm}$ ) who were prescribed laparoscopy for tubal ligation. All individuals were of childbearing age (25-35 years) and had normal menstrual cycles. These women were not given hormone therapy or treatment with gonadotropin-releasing hormone $(\mathrm{GnRH})$ agonists for at least 3 months pre- and post-operatively. No history of autoimmune disease or cancer had been reported for these women. The "thin" endometrial scoring was performed by an expert surgeon on the basis of visual inspection during laparoscopy and patients with an endometrial thickness less than $7 \mathrm{~mm}$ were considered patients with thin endometrium.

\section{Isolation, morphological characterization, and immunophenotyping of MenSCs}

The MenSCs were isolated from menstrual blood specimens collected by participants with a DivaCup (Diva International Inc., Kitchener, ON, USA) on their second day of menstruation and were immediately transported to the laboratory. After separation by Ficoll-Paque (GE Healthcare, Stockholm, Sweden), the mononuclear cells were cultured for 2 weeks to obtain the MenSCs whose confirmation was made by their negativity for cytokeratin (Figure S1). The MenSCs used in all experiments were passages 2 to 9. The morphological differences between TE-MenSCs (MenSCs from patients with a "thin" endometrium) and NTE-MenSCs (MenSCs from patients with a "non-thin" endometrium) in 2D cultures of NTEMenSCs $(n=6)$ and TE-MenSCs $(n=6)$ were examined by phase contrast microscopy. Analysis was performed by 2 reviewers ( $>90 \%$ concordance) who investigated cell circularity using ImageJ software (version 1.42I; https:// imagej.nih.gov.ij/).

\section{Cytokeratin 7 immunofluorescence}

The MenSCs $\left(5 \times 10^{3}\right)$ were cultured in 8 -well chamber slides (Falcon, NJ, USA) for 24 h. Next, the MenSCs were harvested and fixed for $30 \mathrm{~min}$ in $4 \%$ paraformaldehyde. After permeabilization for $10 \mathrm{~min}$ with ice-cold methanol, a mixture of PBS-1\% bovine serum albumin (BSA) was used for saturation reaction. Afterward, the cells were treated with anti-CK7 Alexa Fluor 555 Conjugate (1:50 Abcam, \#ab209601). Then, DAPI was employed to counterstain the cell nuclei. The confocal microscopy (Nikon, Lipsi, Tokyo, Japan) was used for examination of the slides at $40 \times$ magnification and imaging.

\section{Adipogenic induction and differentiation}

Previous studies have demonstrated the adipogenic differentiation potential of MenSCs (29), thus we assessed the adipogenic differentiation potential of MenSCs. For the induction of adipogenic differentiation, dexamethasone (Sigma-Aldrich, St. Louis, MO, USA), 3-isobutyl-1methyl-xanthine (Sigma-Aldrich, USA), and recombinant human insulin (Sigma-Aldrich, USA) and rosiglitazone (Osvah Pharmaceutical Company, Tehran, Iran) were purchased and used for the treatment of MenSCs which were cultured for a period of 7 days. On the third day of culture in Dulbecco's modified Eagle medium (DMEM)-F12 to which fetal bovine serum (FBS) had been added, the MenSCs were collected and cultured in a new DMEM-F12 medium containing FBS, recombinant human insulin, dexamethasone, and indomethacin until the 14th day. Exposure of the differentiated MenSCs was performed by staining the fatty vacuoles with $\mathrm{O}$ red oil which was provided by Sigma Chemicals (St. Louis, MO, USA). The aforementioned methods were applied to the undifferentiated cells which were used as control cells.

\section{Osteogenic induction and differentiation}

Previous studies have demonstrated the osteogenic differentiation potential of MenSCs (29), thus we assessed the osteogenic differentiation potential of MenSCs. MenSCs $\left(4 \times 10^{4}\right.$ cells/well) were cultured in 12 -well plates. The induction of osteogenic differentiation was initiated when cells reached sub-confluence by adding the osteogenic medium. The osteogenic medium was the DMEM medium supplemented with high glucose $(4.5 \mathrm{~g} / \mathrm{L})$ (Gibco, Life Technologies), $10 \%$ FBS, $50 \mu \mathrm{g} / \mathrm{mL}$ ascorbic acid, $1 \%$ penicillin/streptomycin, $10 \mathrm{mmol} / \mathrm{L} \beta$-glycerophosphate (Santa Cruz), and $0.1 \mu \mathrm{mol} / \mathrm{L}$ dexamethasone for 21 days. Alizarin Red S (Carl Roth) staining was subsequently 
performed and osteogenic differentiation was evaluated by microscopic observation. Undifferentiated cells were used as controls and the experiments were performed in triplicates.

\section{Flow cytometry of stem cell surface markers}

In order to verify that the primary MenSCs isolated and used in the experiments were stem cells, MenSCs were used to prepare a cell suspension to which specific labeled antibodies against CD34, CD38, CD44, CD72, CD90, CD105, and SSEA4 described above were added, and after incubation in the dark, the cells were collected for analysis by flow cytometry with the Partec platform (Partec, Münster, Germany). The isotypes were used as negative controls. FlowJo software (version 7.6.1; https://www. flowjo.com/) was used for data processing.

\section{Detection of the proliferation capacity of MenSCs}

The MenSCs $\left(1 \times 10^{4}\right.$ cells/well) that were serum-deprived overnight were cultured in 96-well plates, each well containing $100 \mu \mathrm{L}$ of DMEM culture without phenol red (Gibco, Waltham, MA, USA) but with penicillinstreptomycin solution $(50 \mathrm{U} / \mathrm{mL})$ (Sigma), L-glutamine (2 mM) (Sigma), and FBS (10\%) (Gibco) in a humidified incubator $\left(5 \% \mathrm{CO}_{2}\right)$ over 3 days. Cell proliferation was measured using Cell Counting Test Kit-8 (CCK-8; SigmaAldrich, USA) strictly following the manufacturer's instructions.

\section{Cocultures of MenSCs and human endometrial epithelial cells}

For the co-culture of MenSCs and endometrial epithelial cells (EECS; MenSC/EECS co-cultures), the Transwell culture system was used. The EECS were purchased form Procell Life Science and Technology Co., Ltd. (Cat \#CPH058). Briefly, inoculation of MenSCs $\left(2 \times 10^{5}\right.$ NTEMenSCs or TE-MenSCs cells) was performed in the lower Transwell chambers containing Roswell Park Memorial Institute (RPMI)-1640 medium supplemented with $10 \%$ FBS, while inoculation of EECs obtained from normal individuals was performed in the upper chambers. For the control cultures, the culture of the EECs was performed in the absence of MenSCs. The TE-MenSCs and NTEMenSCs were collected by centrifugation after $72 \mathrm{~h}$ of culture for use in subsequent experiments. In some cases, cells were treated with epidermal growth factor (EGF). For the determination of cytokine concentration, supernatants from the co-cultures were collected. Autologous EECs were used in all experiments.

\section{Real-time quantitative polymerase chain reaction}

The NTE-MenSCs and TE-MenSCs recovered from MenSCs/EECs co-cultures were tested for the expression of EGF, PDGF, FGF, MMP3, and TNF- $\alpha$ using real-time quantitative polymerase chain reaction (qRT-PCR). Total RNA was extracted (Invitrogen, Carlsbad, CA, USA) and used for complementary DNA (cDNA) synthesis which was used as a template for qRT-PCR. The primer sequences for qRT-PCR are presented in Table 1. Amplification was performed under the following reaction conditions: $20 \mu \mathrm{L}$ PCR mixture containing $10 \mu \mathrm{L} 2 \times$ PCR buffer (Takara, Shiga, Japan), $1 \mu \mathrm{L}$ cDNA, $0.4 \mu \mathrm{L}$ primers' $0.8 \mu \mathrm{L}$ TaqMan probe (Operon, Sweden), $0.4 \mu \mathrm{L}$ Dye II $50 \times$ (Takara), and $7 \mu \mathrm{L}$ distilled water autoclave. After incubation at $95{ }^{\circ} \mathrm{C}$ for $10 \mathrm{~s}$, amplification for EGF, PDGF, FGF, MMP3, and TNF- $\alpha$ was conducted at $95^{\circ} \mathrm{C}$ for $5 \mathrm{~s}$ and $60^{\circ} \mathrm{C}$ for $34 \mathrm{~s}$ for 40 cycles. Standardization was performed with reference to the expression of the gene $\beta$-Actin. The experiments were performed in triplicate on the ABI Prism 7500 platform (Applied Biosystems, USA). The $\Delta \Delta \mathrm{Ct}$ method was used to calculate the relative expression of the studied genes.

\section{Western blot}

The NTE-MenSCs and TE-MenSCs were recovered from the MenSCs/EECs co-cultures and homogenized in lysis buffer on ice by vortexing every $5 \mathrm{~min}$ for 15 min. Subsequently, after centrifugation at $12,000 \mathrm{~g}$ for $15 \mathrm{~min}$, the supernatants were collected. Then, the protein concentration of the samples was detected by the bicinchoninic acid (BCA) method using an appropriate kit (Pierce, Rockford, IL, USA), followed by protein separation by $10 \%$ sodium dodecyl sulfate-polyacrylamide gel electrophoresis (SDS-PAGE; Sigma, USA) and transfer to a polyvinylidene fluoride (PVDF) membrane $(0.45 \mu \mathrm{m})$ purchased from Millipore (Burlington, MA, USA). Subsequently, blocking was performed at room temperature in a buffer solution $[0.05 \%$ phosphate-buffered saline (PBS)-Tween 20] containing 5\% skim milk powder for $2 \mathrm{~h}$. Then, after washing, the membranes were incubated overnight at $4{ }^{\circ} \mathrm{C}$ with primary antibodies against p21 Ras, p38 MAPK, and $\beta$-Actin. After washing, the membrane 
Table 1 Primers used in RT-qPCR experiments.

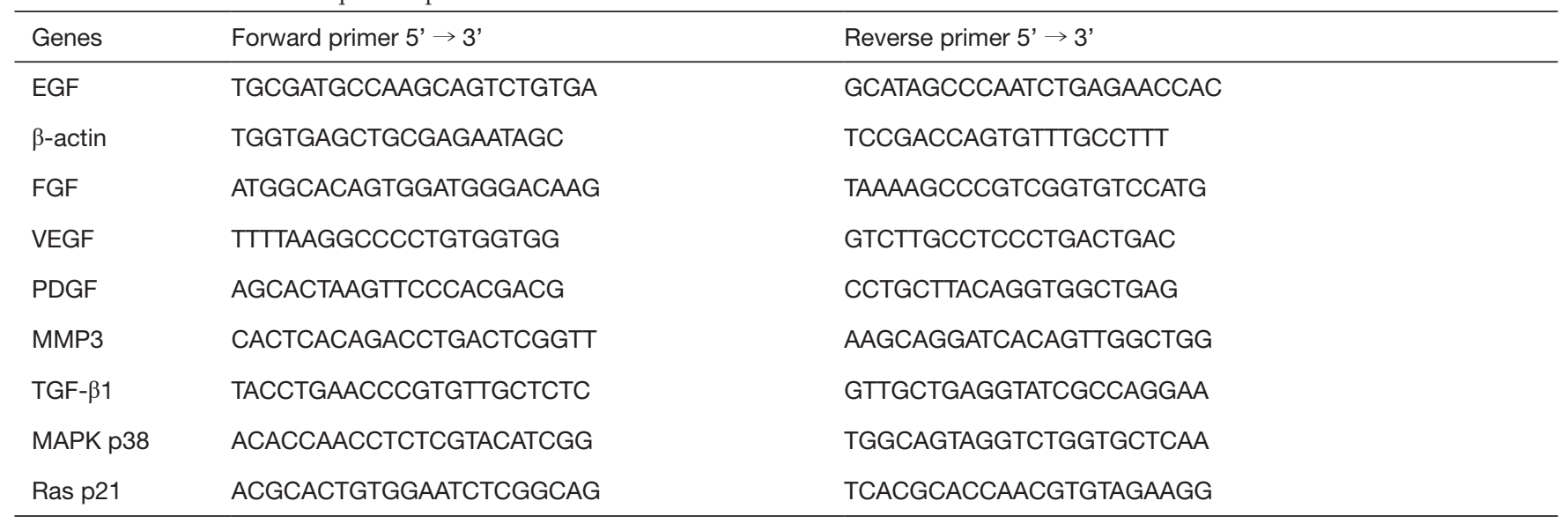

was probed with secondary antibodies conjugated to horseradish peroxidase $(1: 9,000)$ at room temperature over a period of $1 \mathrm{~h}$ followed by successive washes with PBSTween 20. Finally, visualization was done with the enhanced chemiluminescence (ECL) detection kit (GE Healthcare, Chicago, IL, USA) according to the vendor's manual and ImageJ software was used for densitometric quantification of the bands for the calculation of relative protein expression to $\beta$-Actin.

\section{Cytokine assays}

Supernatants from MenSCs/EECs co-cultures were enzyme-linked immunosorbent assay (ELISA)-tested using commercially available EGF, PDGF, FGF, MMP3, VEGF, and TGF- $\beta 1$ ELISA kits (R\&D systems, USA). The optical density of the wells was measured at $450 \mathrm{~nm}$ using spectrophotometric methods with microplate readers.

\section{Statistical analysis}

Statistical differences were evaluated by the one-way analysis of variance (ANOVA) test using GraphPad Prism software (version 6; GraphPad Software, San Diego, CA, USA). Differences were considered significant when $\mathrm{P} \leq 0.05$.

\section{Results}

\section{Characterization of NTE-MenSCs and TE-MenSCs}

In order to see if there are morphological differences between NTE-MenSCs and TE-MenSCs, microscopic examinations were performed. The results indicated that the NTE-MenSCs were like fibroblasts more or less fusiform (Figure 1A), while the TE-MenSCs were more or less spherical with greater circularity (Figure $1 A$ ). In addition, TE-MenSCs showed their ability to form small colonies (Figure $1 B$ ); the population doubling (PD) of NTE-MenSCs was greater than that of the TE-MenSCs $(\mathrm{P}<0.01)$ over the cell expansion through passages 1-9 (Figure 1B).

In order to examine the ability of MenSCs to differentiate into adipocytes, these cells were stained with O-red oil. As shown in Figure 1C, the formation of oil droplets in cells that had differentiated into adipocytes was observed. In the osteogenesis induction medium, we also observed that the TE-MenSCs and NTE-MenSCs were all capable of osteogenic differentiation, and the osteogenic differentiation capacity of NTE-MenSCs was greater than that of TE-MenSCs (Figure 1C). No staining was observed for undifferentiated control MenSCs (Figure 1C).

Flow cytometry showed that TE-MenSCs and NTEMenSCs were positive for CD90, CD34, CD72, and CD44, but negative for CD38, SSEA-4, and CD105 (Figure 2A,2B). Our results showed that the expression [mean fluorescence intensity (MFI)] of CD90, CD34, CD72, and CD44 was significantly lower in NTE-MenSCs than in TE-MenSCs (Figure 2A,2B).

\section{Effect of NTE-MenSCs and TE-MenSCs on the proliferation, invasion, and adbesion of EECs}

In order to detect the effect of MenSCs on EECs, we cocultured EECs in the presence of NTE-MenSCs and TEMenSCs in Transwell chambers or in the presence of their 
A
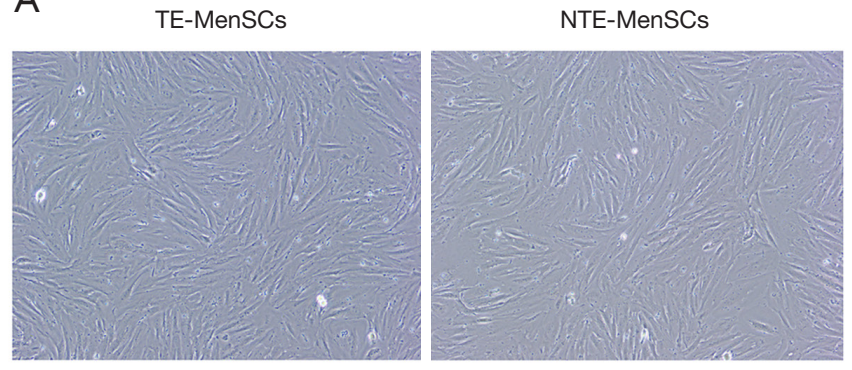

B

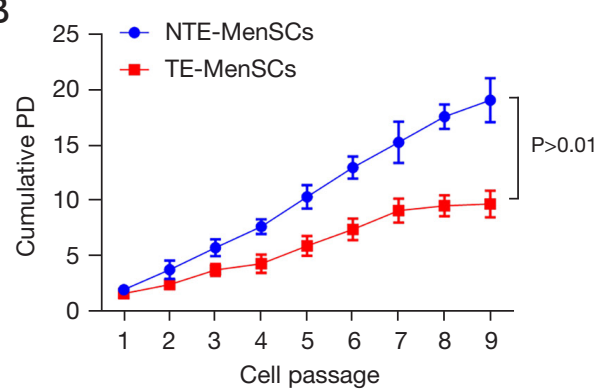

C
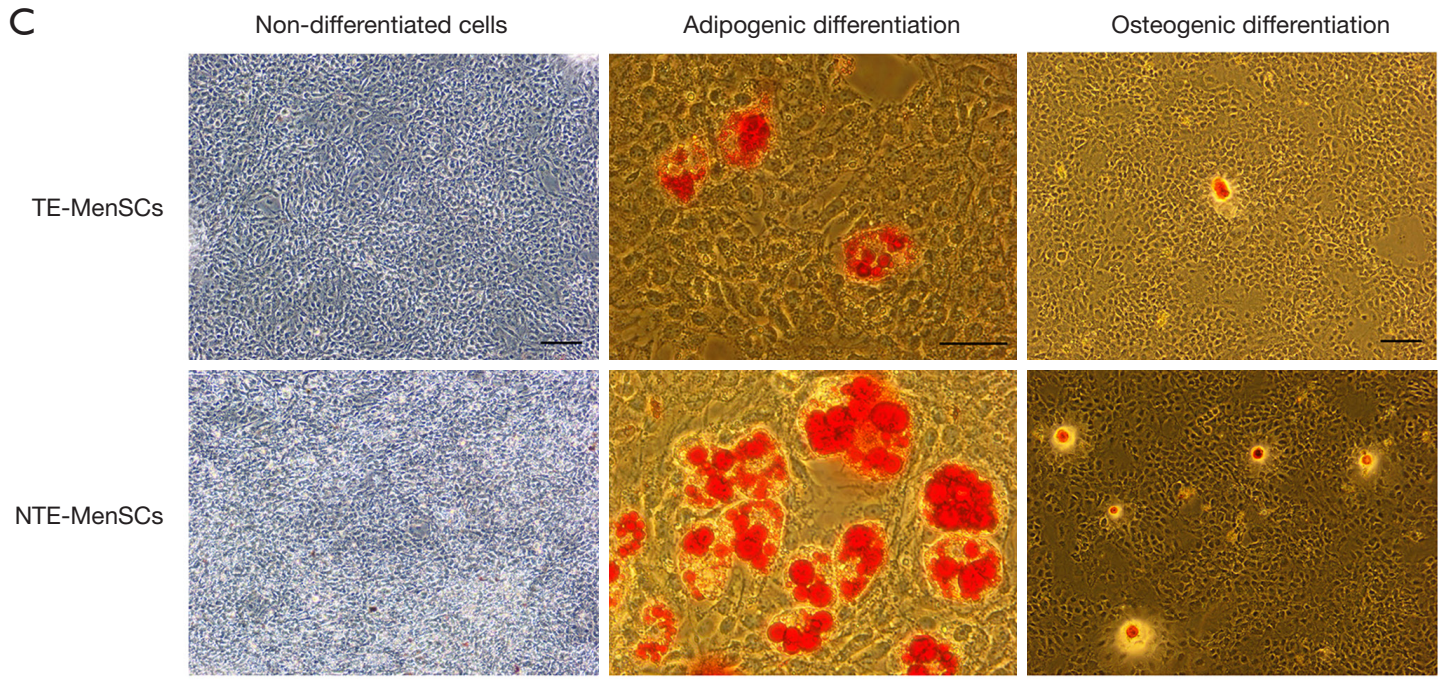

Figure 1 Characteristics of TE-MenSCs and NTE-MenSCs. (A) Morphological analysis of NTE-MenSCs and TE-MenSCss cultures by microscopic examination (×400); (B) colony-forming ability of NTE-MenSCs and TE-MenSCs; (C) adipogenic differentiation and osteogenic differentiation of TE-MenSCs and NTE-MenSCs was detected by red oil staining $\mathrm{O}$ and induction in osteogenic medium, respectively. Staining of undifferentiated cells was used as a control group $(x 400$, scale bar $=50 \mu \mathrm{m})$. TE-MenSCs, menstrual stem cells from thin endometrium; NTE-MenSCs, menstrual stem cells from non-thin endometrium.

supernatants. The results showed that TE-MenSCs or their supernatant (TE-MenSCs CM) had an inhibitory effect on the proliferation of EECs compared to NTE-MenSCs or NTE-MenSCs CM (Figure 3A). Cell cycle analysis by flow cytometry indicated that TE-MenSCs or their supernatant increased the proportion of cells in G2-phase, indicating their potential effect on cell cycle arrest compared to NTEMenSCs, NTE-MenSCs CM, or EECs alone groups (Figure 3B). No significant effect was observed on the action of TE-MenSCs and their supernatant on EEC apoptosis compared to NTE-MenSCs, NTE-MenSCs $\mathrm{CM}$, or EECs alone groups in the Tunel experiments (Figure 3C). Compared to NTE-MenSCs, TE-MenSCs and their supernatant significantly increased invasive ability of EECs $(\mathrm{P} \leq 0.05)$ (Figure 4).

\section{Effects of MenSCs and their supernatants on gene expression}

The qRT-PCR experiments for EGF, PDGF, FGF, MMP3, VEGF, and TGF- $\beta 1$ genes were performed after coculturing EECs in the presence of NTE-MenSCs or TEMenSCs and their superntants from transwell MenSCs/ ECCs co-cultures. As shown in Figure 5, the expression levels of EGF, PDGF, FGF, MMP3, VEGF, and TGF- $\beta 1$ were more promoted in EECs incubated in the presence of TE-MenSCs or their supernatant compared to NTEMenSCs or their supernatant $(\mathrm{P} \leq 0.001)$ (Figure 5). The ELISA test also confirmed the significantly increased release of proteins encoded by these genes in the supernatant of TE-MenSCs and NTE-MenSCs compared to the supernatant of EECs (Figure 6). These results suggested 
A TE-MenSCs
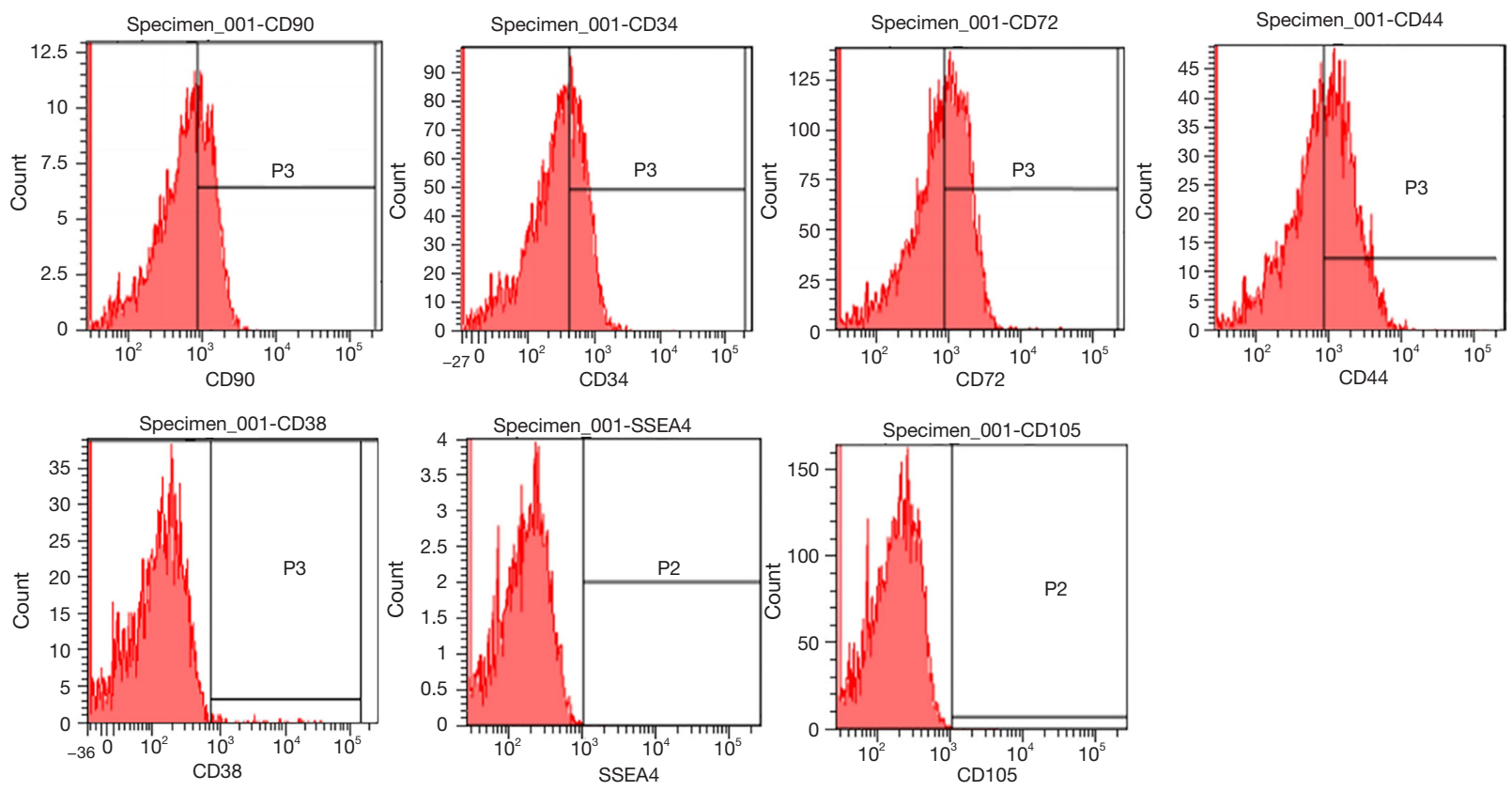

B NTE-MensCs
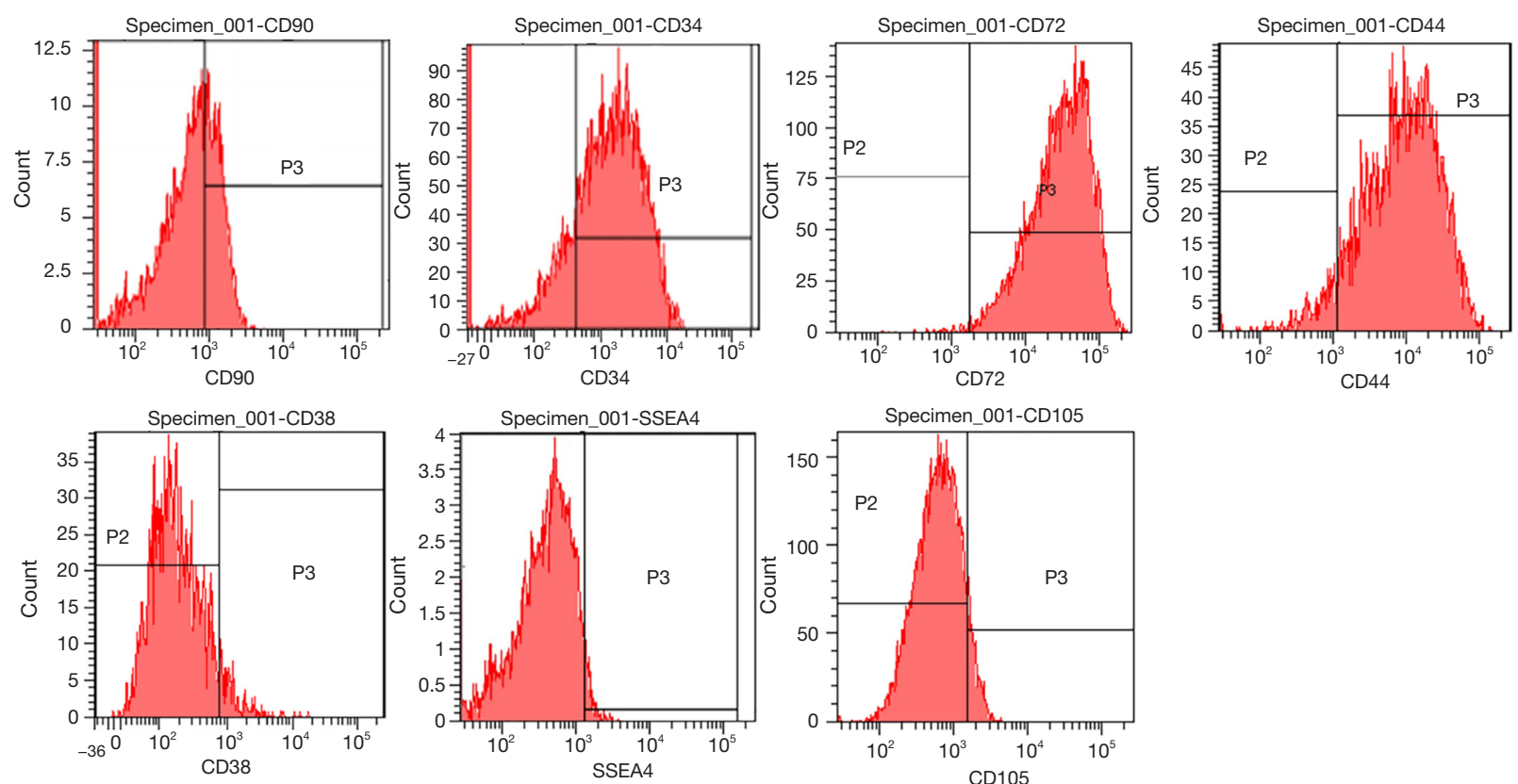

Figure 2 Detection of stem cell membrane markers of NTE-MenSCs and TE-MenSCs by flow cytometry. (A) Flow cytometry images for TE-MenSCs; (B) flow cytometry images for NTE-MenSCs. TE-MenSCs, menstrual stem cells from thin endometrium; NTE-MenSCs, menstrual stem cells from non-thin endometrium. 
A

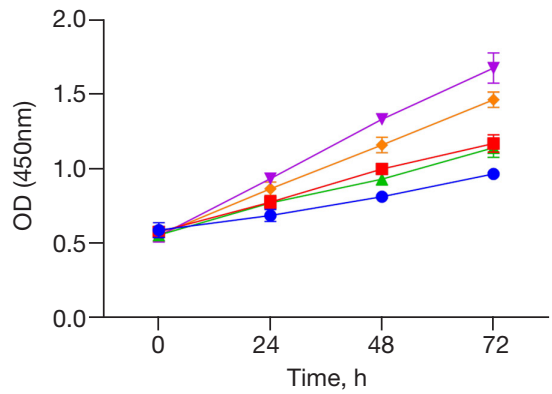

- EECs

- EECs + TE-MenSCs

$\_$EECs + TE-MenSCs CM

$\checkmark$ EECs + NTE-MenSCs

$\rightarrow$ EECs + NTE-MenSCs CM
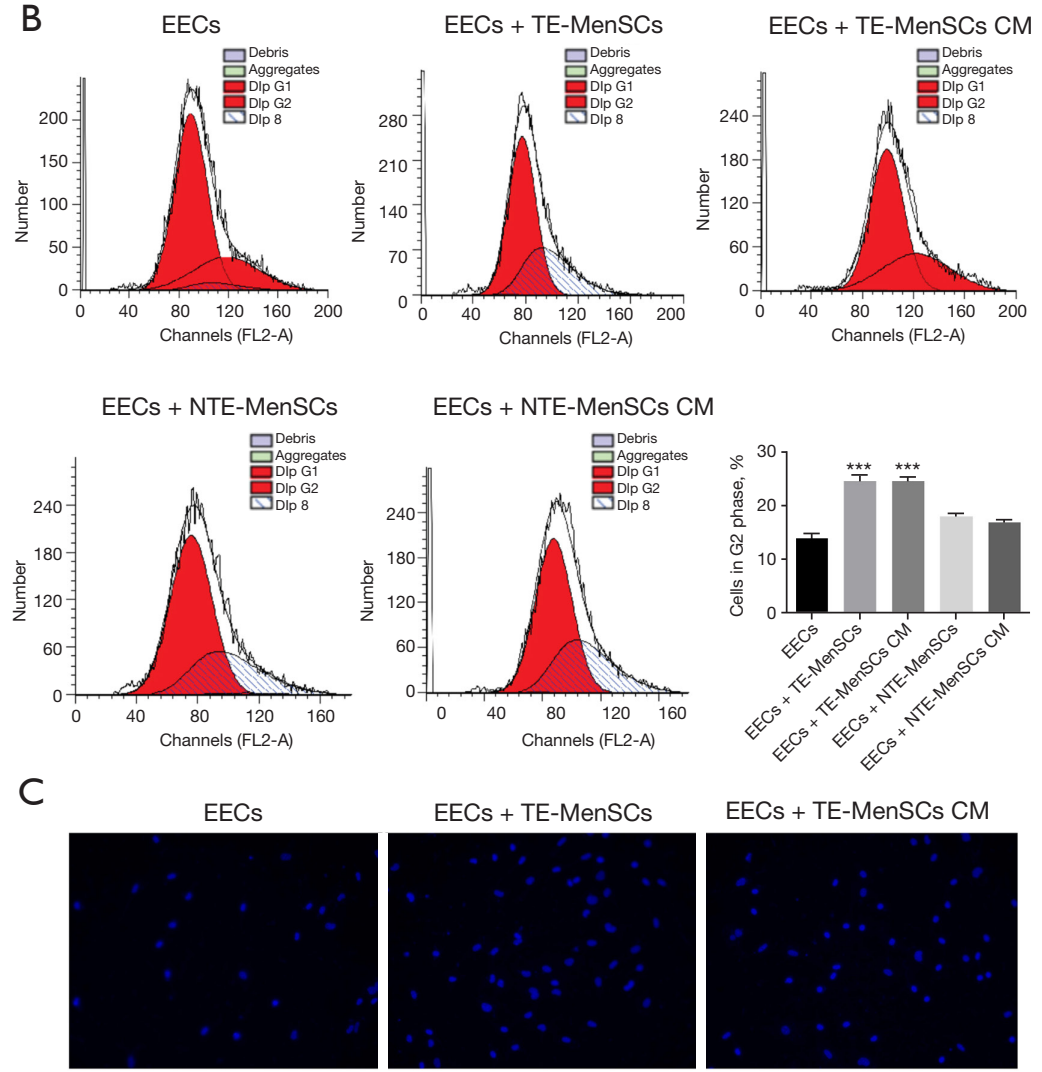

EECs + TE-MenSCs

EECs + TE-MenSCs CM
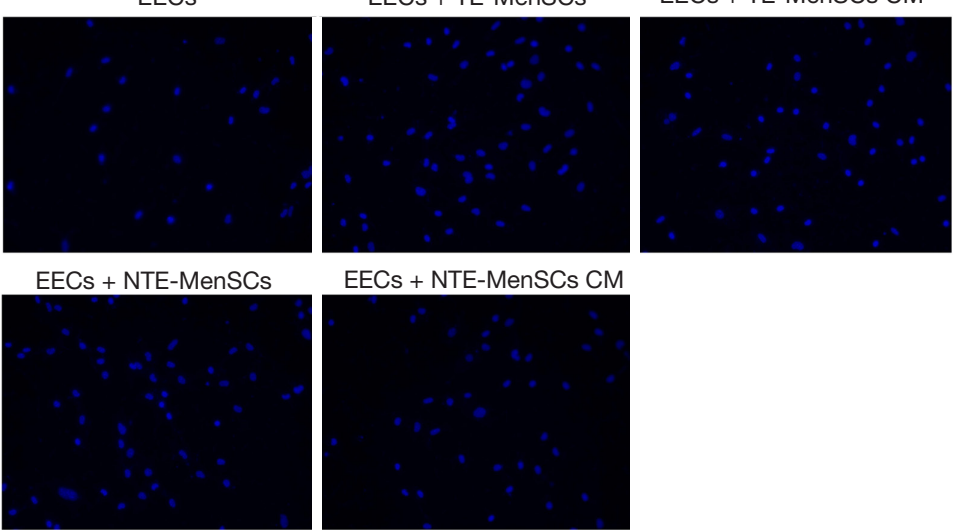

EECs + NTE-MenSCs CM

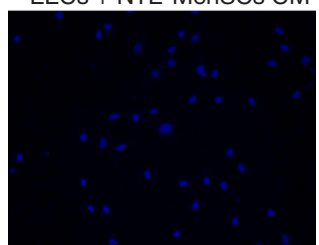

Figure 3 Proliferation, cell cycle and apoptosis analysis of EECs co-cultured with NTE-MenSCs and TE-MenSCs or in the presence of their supernatant. The potential for proliferation, cell cycle and apoptosis EECs cocultured with NTE-MenSCs and TE-MenSCs was measured using cck8, flow cytometry and Tunel assay, respectively. (A) Optical density indicating the proliferation of EECs as determined by CCK8; (B) flow cytometry images of the cell cycle and quantification of cells in G2 phase; (C) Tunel assay images of cell apoptosis of EECs $(\times 400) .{ }^{* *} \mathrm{P} \leq 0.0001$ compared to EECs group. TE-MenSCs, menstrual stem cells from thin endometrium; NTE-MenSCs, menstrual stem cells from non-thin endometrium; EECs, endometrial epithelial cells. 

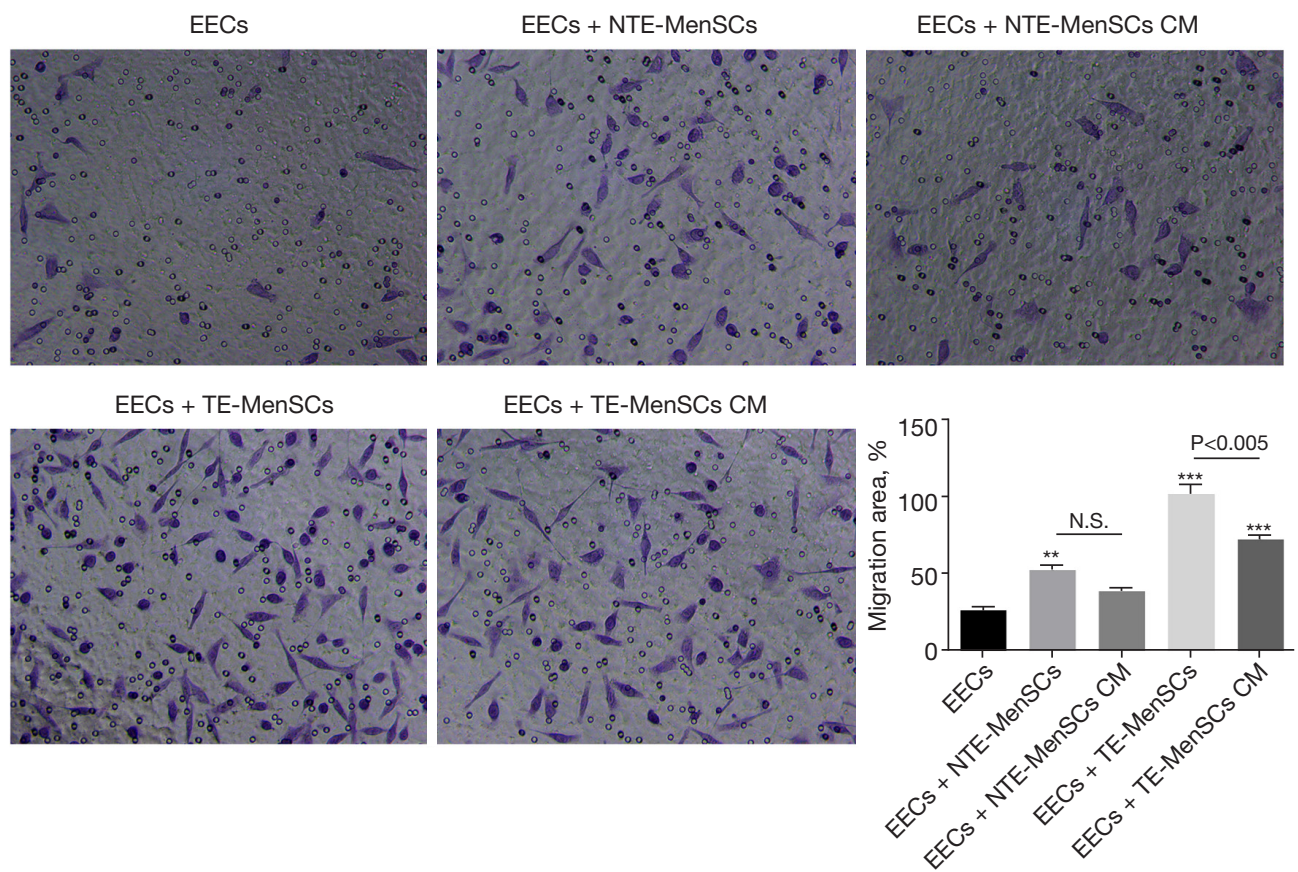

Figure 4 Invasion of EECs in the presence of NTE-MenSCs and TE-MenSCs or their supernatants. Cell invasion was determined using the matrigel cell invasion experiment in transwell chambers $(\times 400)$. Compared to EECs group, ${ }^{* *} \mathrm{P} \leq 0.01$; compared to EECs group, ${ }^{* * *} \mathrm{P} \leq 0.001$. NS, non-significant among the indicated groups. TE-MenSCs, menstrual stem cells from thin endometrium; NTE-MenSCs, menstrual stem cells from non-thin endometrium; EECs, endometrial epithelial cells.

that TE-MenSCs may influence the phenotypes of EECs by regulating these proteins.

\section{TE-MenSCs regulate the phenotype of EECs by activating the EGF/Ras p21 pathway}

To explore the mechanism underlying the effect of TEMenSCs on EECs, we chose to investigate the effect of EGF on the phenotypes of EECs as this gene was the most induced by the TE-MenSCs. The results showed that the treatment of EECs by EGF significantly increased the proliferation and invasiveness of EECs (Figure $7 A, 7 B$ ). Moreover, the expression of Ras p21 was increased by EGF treatment both at the messenger RNA (mRNA) and protein levels while MAPK p38 expression was increased only at mRNA level (Figure 7C,7D), suggesting that the effect of TE-MenSCs may be partially driven by its activation of the EGF/Ras p21 pathway.

\section{Discussion}

Low endometrial receptivity is a condition frequently encountered in women of reproductive age, which has great influence on the embryo implantation, and occurs in women with thin endometrium $(1,8)$. The application of stem cells in the treatment of various diseases has been proven $(13,14,18,24)$; however, the therapeutic effect of stem cells in women with thin endometrium is ill-defined. In the present work, we extracted MenSCs from healthy women and women with thin endometrium in order to explore their effects on the invasiveness, proliferation, and angiogenesis of epithelial endometrial cells (EECs). After their characterization, our results showed that the MenSCs from women with thin endometrium were able to induce the proliferation and invasion of EECs but had no effect on apoptosis. Further coculture experiments indicated that TE-MenSCs supernatant had high levels of cytokines and both TE-MenSCs and their supernatants were able to stimulate the expressions of EGF, PDGF, FGF, MMP3, VEGF, and TGF- $\beta 1$. The effect of TE-MenSCs was likely to be driven through its activation of the EGF/ Ras p21 pathway. These findings pave the way for the application of MenSCs in the treatment of low endometrial receptivity in women. In the present study, surface markers 

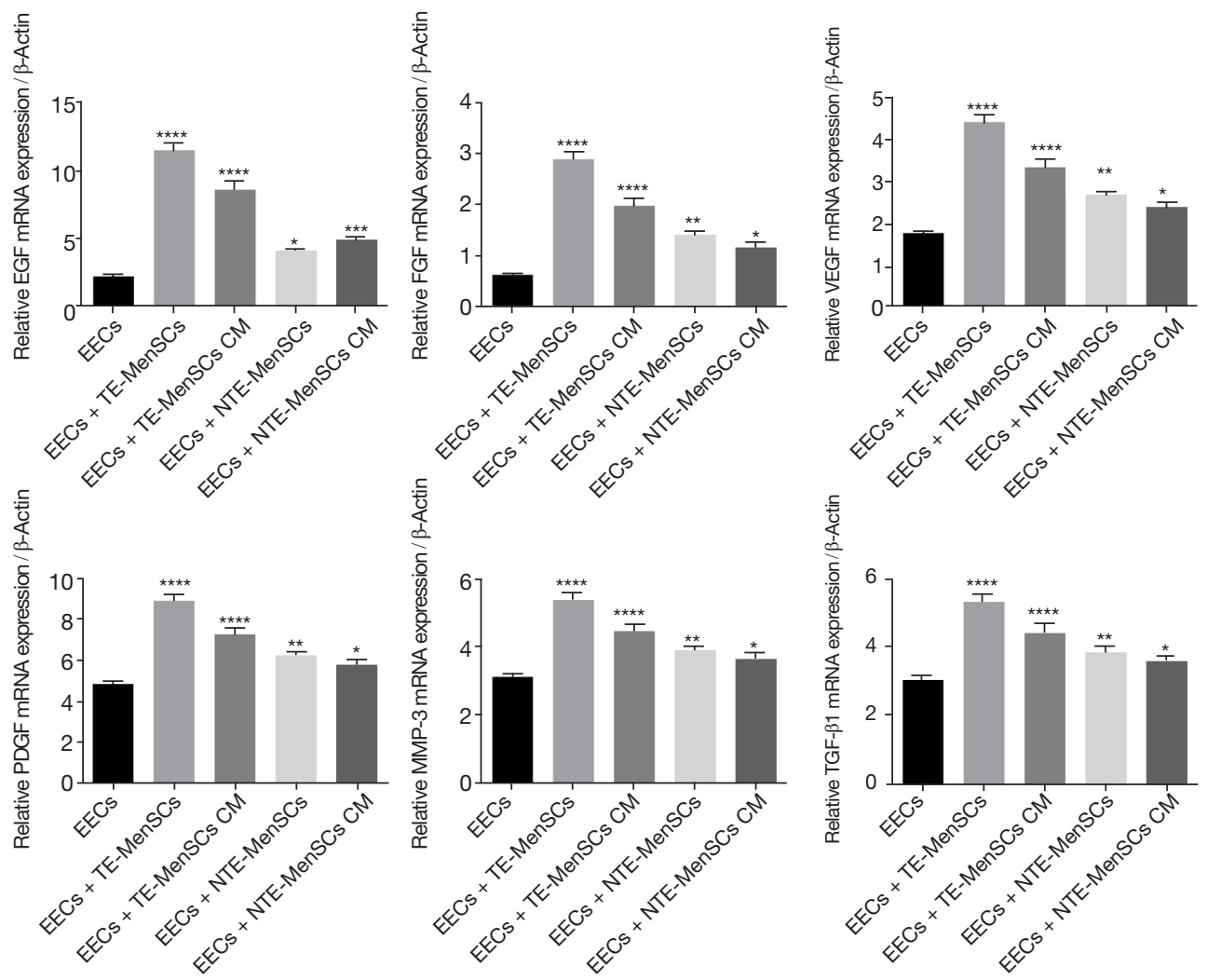

Figure 5 Quantitative evaluation of the mRNA expression of EGF, PDGF, FGF, MMP3, VEGF and TGF- $\beta 1$ genes in EECs co-cultured in the presence of NTE-MenSCs, TE-MenSCs or their supernatant. Gene expression was determined by qRT-PCR. *P $\leq 0.05$; ${ }^{* *} \mathrm{P} \leq 0.01$; ${ }^{* * *} \mathrm{P} \leq 0.001 ;{ }^{* * *} \mathrm{P} \leq 0.0001$ compared to EECs group. TE-MenSCs, menstrual stem cells from thin endometrium; NTE-MenSCs, menstrual stem cells from non-thin endometrium; EECs, endometrial epithelial cells; qRT-PCR, real-time quantitative polymerase chain reaction.

of MenSCs were CD90, CD34, CD72, and CD44, which corroborated previous findings (27) and confirmed the stemness of MenSCs. These results also corroborated with the studies indicating that MenSCs have some commons markers with MSC surface markers such as CD90, CD73, and CD105 but differ markers such CD49a, OCT-4 and SSEA-4 which are found expressed in MenSCs and STRO1 which is not expressed in MenSCs (26). Morphological differences were also observed between the NTE-MenSCs and the TE-MenSCs, which also corroborated with the above work. These differences are probably due to the pathological microenvironment of the endometrium and are undoubtedly responsible for the differences in the response of EECs to both types of MenSCs.

Our study indicated that TE-MenSCs were able to stimulate the invasiveness and proliferation of EECs and regulate the levels of cytokines. These results indicated that TE-MenSCs stimulate inflammatory pathways to change the function of EECs, which may impact on embryo implantation in vivo. Thus, by manipulating the phenotype of MensCs, we can module the phenotypes of EECs in women, which is of great value in dealing with embryo implantation in women with thin endometrium. Our findings were in line with previous studies indicating the ability of MenSCs to regulate the proliferation, wound healing, migration, and apoptosis of endometrial stromal cells (30).

Human endometrial tissue and primary stromal cell culture contain immunoreactive EGF, PDGF-AB as well as EGF and PDGF-beta receptors. Both EGF and PDGF are mitogenic for endometrial stromal cells, suggesting an autocrine/paracrine role in modulation of endometrial cell growth and differentiation. Herein, we found that EGF, PDGF, and FGF were significantly regulated by the TEMenSCs, which further indicated that the TE-MenSCs might regulate the immune response of EECs. In addition, 

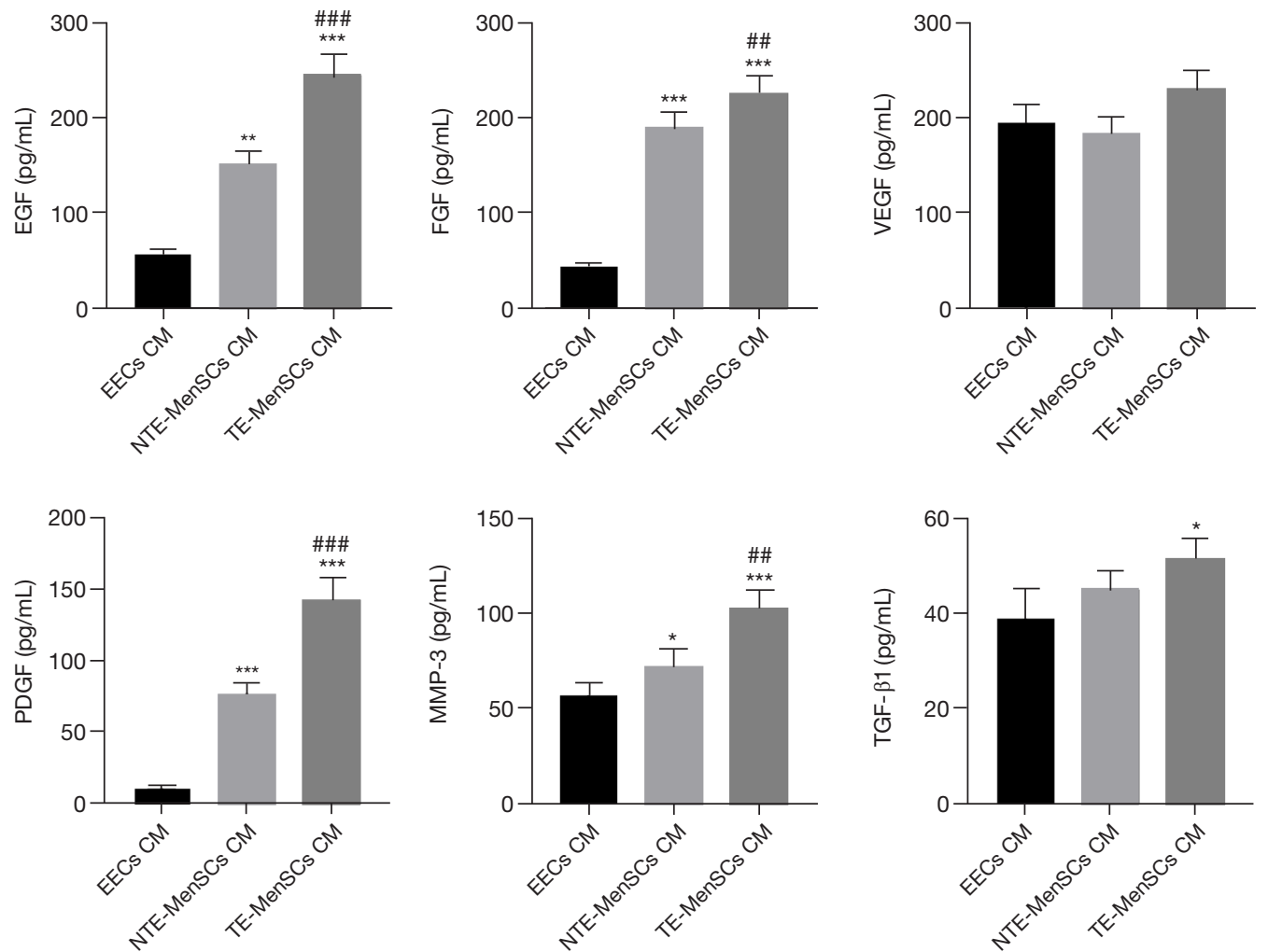

Figure 6 Determination of protein concentrations. The proteins levels of EGF, PDGF, FGF, MMP3, VEGF, and TGF- $\beta 1$ proteins in the supernatant of EECs, TE-MenSCs and NTE-MenSCs was determined by ELISA. ${ }^{*} \mathrm{P} \leq 0.05,{ }^{* *} \mathrm{P} \leq 0.01$, ${ }^{* *} \mathrm{P} \leq 0.001$, compared to EECs group; ${ }^{\#} \mathrm{P} \leq 0.01 ;{ }^{\# \#} \mathrm{P} \leq 0.001$, compared to NTE-MenSCs group. TE-MenSCs, menstrual stem cells from thin endometrium; NTE-MenSCs, menstrual stem cells from non-thin endometrium; EECs, endometrial epithelial cells; ELISA, enzyme-linked immunosorbent assay.

A

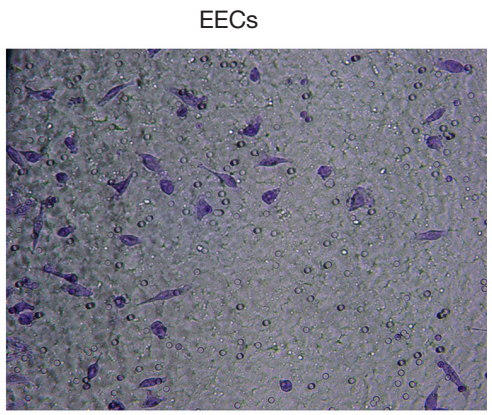

$\mathrm{EECs}+\mathrm{EGF}$

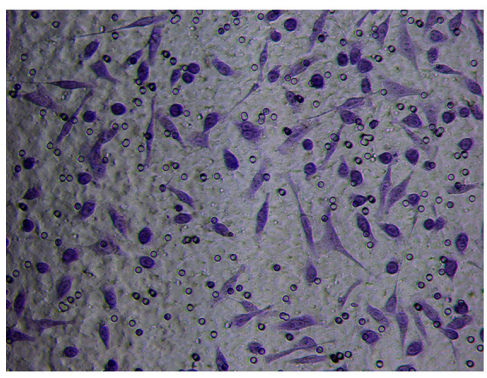

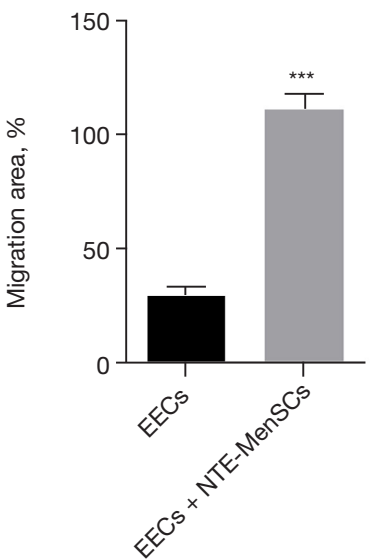



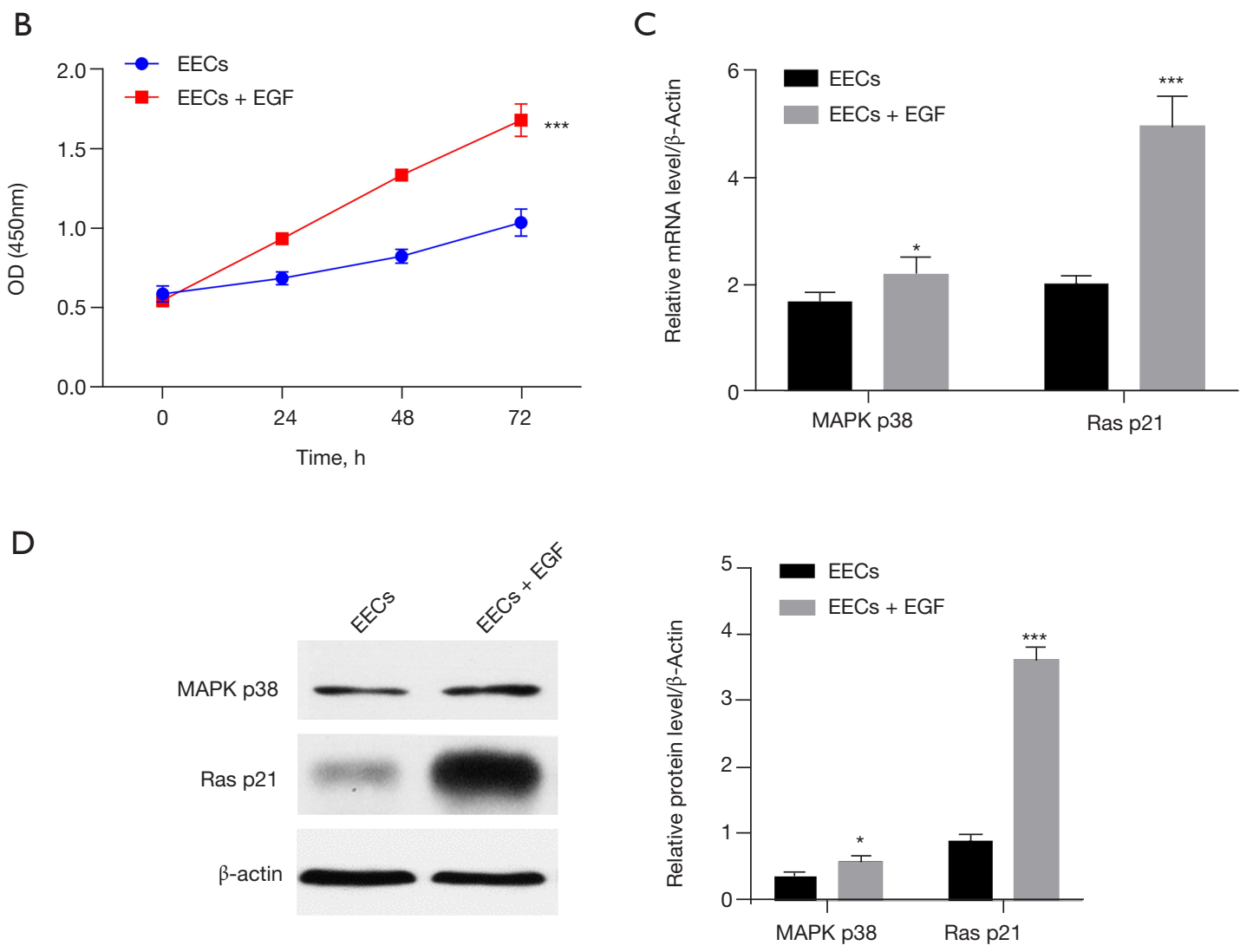

Figure 7 TE-MenSCs regulates the phenotype of EECs by activating the EGF/Ras p21 pathway. (A) Effect of EGF treatment on cell invasion of EECs. (B) Effect of EGF treatment on cell proliferation of EECs ( $\times 400)$. (C) Effect of EGF treatment on the mRNA expression of MAPK p38 and Ras p21 in EECs as determined by RT-PCR. (D) Effect of EGF treatment on the protein expression of MAPK p38 and Ras p21 in EECs as determined by western blotting. TE-MenSCs, menstrual stem cells from thin endometrium; NTE-MenSCs, menstrual stem cells from non-thin endometrium; EECs, endometrial epithelial cells; mRNA, messenger RNA; RT-PCR, reverse transcription polymerase chain reaction.

these proteins are reported to play a significant role in the vascularized adipose. Thus, our results indicated that TEMenSCs can also regulate vascularization of EECs, which might be very detrimental for embryo implantation in vivo. Furthermore, the expression of MMP3 was also influenced by the TE-MenSCs, which is indicative of TE-MenSCs influence on the extracellular matrix and fibrosis. Our results also showed that the EGF/Ras p21 axis is partially involved in the mechanism underlying the effect of TEMenSCs on the proliferation and invasiveness of EECs.

Conclusively, our study showed for the first time that the MenSCs can regulate the phenotypes of EECs by governing important biological processes. These findings are vitally important to the treatment of thin endometrium for embryo implantation. Further in vivo experiments are needed for further validation of these findings.

\section{Acknowledgments}

Funding: This study was funded by grants from the National Key Research and Development Plan of China (Study on the Regeneration Mechanism of Human Epithelial Tissue; 2017YFA0104603) and Shanghai Natural Science Fund (21ZR1450700).

\section{Footnote}

Reporting Checklist: The authors have completed the MDAR reporting checklist. Available at https://dx.doi. org/10.21037/atm-21-4652

Data Sharing Statement: Available at https://dx.doi. org/10.21037/atm-21-4652 
Conflicts of Interest: All authors have completed the ICMJE uniform disclosure form (available at https://dx.doi. org/10.21037/atm-21-4652). The authors have no conflicts of interest to declare.

Ethical Statement: The authors are accountable for all aspects of the work in ensuring that questions related to the accuracy or integrity of any part of the work are appropriately investigated and resolved. This study was approved by the Institutional Review Board and the Ethics Committee of Tongji University (2019tjdx352), and all participants had signed an informed consent form before the start of the study. All procedures performed in this study involving human participants were in accordance with the Declaration of Helsinki (as revised in 2013).

Open Access Statement: This is an Open Access article distributed in accordance with the Creative Commons Attribution-NonCommercial-NoDerivs 4.0 International License (CC BY-NC-ND 4.0), which permits the noncommercial replication and distribution of the article with the strict proviso that no changes or edits are made and the original work is properly cited (including links to both the formal publication through the relevant DOI and the license). See: https://creativecommons.org/licenses/by-nc-nd/4.0/.

\section{References}

1. Bashiri A, Halper KI, Orvieto R. Recurrent Implantation Failure-update overview on etiology, diagnosis, treatment and future directions. Reprod Biol Endocrinol 2018;16:121.

2. Tanbo T, Fedorcsak P. Endometriosis-associated infertility: aspects of pathophysiological mechanisms and treatment options. Acta Obstet Gynecol Scand 2017;96:659-67.

3. Wurn BF, Wurn LJ, King CR, et al. Treating female infertility and improving IVF pregnancy rates with a manual physical therapy technique. MedGenMed 2004;6:51.

4. Alegre L, Del Gallego R, Bori L, et al. Assessment of embryo implantation potential with a cloud-based automatic software. Reprod Biomed Online 2021;42:66-74.

5. Gardner DK, Lane M, Stevens J, et al. Blastocyst score affects implantation and pregnancy outcome: towards a single blastocyst transfer. Fertil Steril 2000;73:1155-8.

6. Rosen MP, Shen S, Rinaudo PF, et al. Fertilization rate is an independent predictor of implantation rate. Fertil Steril 2010;94:1328-33.
7. Wong KM, van Wely M, Mol F, et al. Fresh versus frozen embryo transfers in assisted reproduction. Cochrane Database Syst Rev 2017;3:CD011184.

8. Mahajan N. Endometrial receptivity array: Clinical application. J Hum Reprod Sci 2015;8:121-9.

9. Lebovitz O, Orvieto R. Treating patients with "thin" endometrium - an ongoing challenge. Gynecol Endocrinol 2014;30:409-14.

10. Zhang T, Li Z, Ren X, et al. Endometrial thickness as a predictor of the reproductive outcomes in fresh and frozen embryo transfer cycles: A retrospective cohort study of 1512 IVF cycles with morphologically good-quality blastocyst. Medicine (Baltimore) 2018;97:e9689.

11. Mahajan N, Sharma S. The endometrium in assisted reproductive technology: How thin is thin? J Hum Reprod Sci 2016;9:3-8.

12. Kim H, Shin JE, Koo HS, et al. Effect of Autologous Platelet-Rich Plasma Treatment on Refractory Thin Endometrium During the Frozen Embryo Transfer Cycle: A Pilot Study. Front Endocrinol (Lausanne) 2019;10:61.

13. Tersoglio AE, Tersoglio S, Salatino DR, et al. Regenerative therapy by endometrial mesenchymal stem cells in thin endometrium with repeated implantation failure. A novel strategy. JBRA Assist Reprod 2020;24:118-27.

14. Azizi R, Aghebati-Maleki L, Nouri M, et al. Stem cell therapy in Asherman syndrome and thin endometrium: Stem cell- based therapy. Biomed Pharmacother 2018;102:333-43.

15. Du J, Lu H, Yu X, et al. Efficacy and safety of plateletrich plasma for the treatment of thin endometrium: A protocol for systematic review and meta-analysis. Medicine (Baltimore) 2020;99:e18848.

16. Liao DY, Liu JH, Zheng YP, et al. OCT proves that vitreomacular adhesion is significantly more likely to develop vision-threatening retinal complications than vitreomacular separation. BMC Ophthalmol 2020;20:163.

17. Liu KE, Hartman M, Hartman A. Management of thin endometrium in assisted reproduction: a clinical practice guideline from the Canadian Fertility and Andrology Society. Reprod Biomed Online 2019;39:49-62.

18. Mouhayar Y, Sharara FI. G-CSF and stem cell therapy for the treatment of refractory thin lining in assisted reproductive technology. J Assist Reprod Genet 2017;34:831-7.

19. Xia L, Meng Q, Xi J, et al. The synergistic effect of electroacupuncture and bone mesenchymal stem cell transplantation on repairing thin endometrial injury in rats. Stem Cell Res Ther 2019;10:244. 
20. Zhao J, Zhang Q, Wang Y, et al. Uterine infusion with bone marrow mesenchymal stem cells improves endometrium thickness in a rat model of thin endometrium. Reprod Sci 2015;22:181-8.

21. Ding DC, Shyu WC, Lin SZ. Mesenchymal stem cells. Cell Transplant 2011;20:5-14.

22. Uccelli A, Moretta L, Pistoia V. Mesenchymal stem cells in health and disease. Nat Rev Immunol 2008;8:726-36.

23. Bozorgmehr M, Gurung S, Darzi S, et al. Endometrial and Menstrual Blood Mesenchymal Stem/Stromal Cells: Biological Properties and Clinical Application. Front Cell Dev Biol 2020;8:497.

24. Chen L, Qu J, Cheng T, et al. Menstrual blood-derived stem cells: toward therapeutic mechanisms, novel strategies, and future perspectives in the treatment of diseases. Stem Cell Res Ther 2019;10:406.

25. Chen L, Qu J, Xiang C. The multi-functional roles of menstrual blood-derived stem cells in regenerative medicine. Stem Cell Res Ther 2019;10:1.

26. Uzieliene I, Urbonaite G, Tachtamisevaite Z, et al. The

Cite this article as: Zhao $M$, Chi $F$, Zhang T, Teng $X$, Li K. Human menstrual blood-derived mesenchymal stem cells regulation of the EGF/Ras p21 pathway as a potential therapeutic target for thin endometrium. Ann Transl Med 2021;9(18):1476. doi: 10.21037/atm-21-4652
Potential of Menstrual Blood-Derived Mesenchymal Stem Cells for Cartilage Repair and Regeneration: Novel Aspects. Stem Cells Int 2018;2018:5748126.

27. Hu J, Song K, Zhang J, et al. Effects of menstrual blood-derived stem cells on endometrial injury repair. Mol Med Rep 2019;19:813-20.

28. Chen L, Guo L, Chen F, et al. Transplantation of menstrual blood-derived mesenchymal stem cells (MbMSCs) promotes the regeneration of mechanical injuried endometrium. Am J Transl Res 2020;12:4941-54.

29. Uzieliene I, Bagdonas E, Hoshi K, et al. Different phenotypes and chondrogenic responses of human menstrual blood and bone marrow mesenchymal stem cells to activin A and TGF- $\beta 3$. Stem Cell Res Ther 2021;12:251.

30. Zhu H, Jiang Y, Pan Y, et al. Human menstrual bloodderived stem cells promote the repair of impaired endometrial stromal cells by activating the p38 MAPK and AKT signaling pathways. Reprod Biol 2018;18:274-81. 


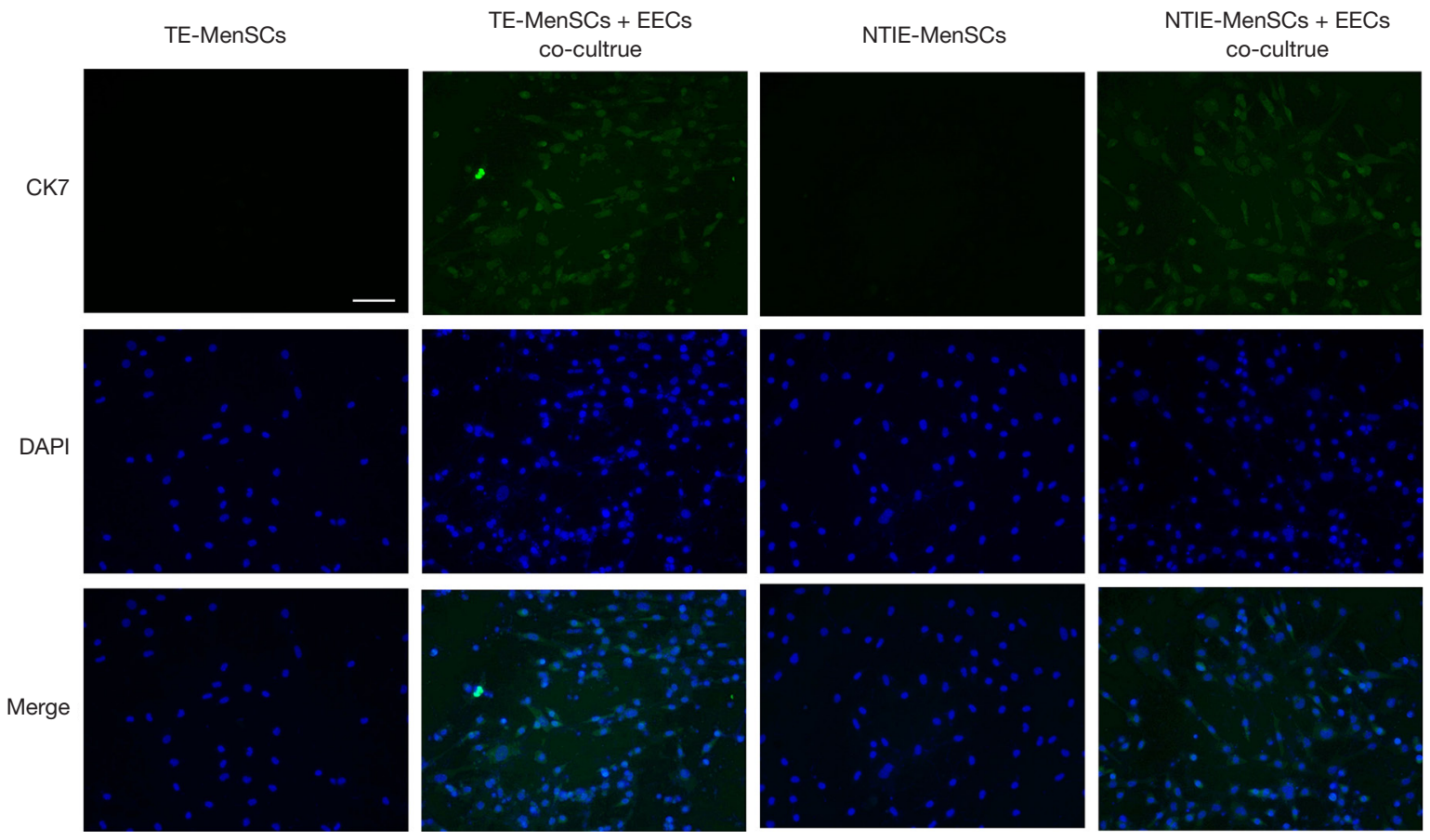

Figure S1 Immunofluorescence detection of CK7 expression $(\times 400)$. Scale bar $=50 \mu \mathrm{m}$. 